\title{
Neue und überraschende Daten
}

Fragestellung: Wie häufig und unter welchen Umständen betreiben Erwerbstätige pharmakologisches Neuroenhancement und hat der Konsum seit 2008 zugenommen?

Hintergrund: Als pharmakologisches Neuroenhancement oder Hirn-Doping bezeichnet man den Gebrauch verschreibungspflichtiger Medikamente ohne medizinische Notwendigkeit durch Gesunde mit den Zielen der Leistungssteigerung oder der Verbesserung des psychischen Wohlbefindens. Das Ziel der Leistungssteigerung wird vor allem mit Stimulanzien wie Methylphenidat, Wachmachern wie Modafinil oder Antidementiva verfolgt, während die Verbesserung des psychischen Wohlbefindens durch Antidepressiva oder Betablocker zum Abbau von Ängsten und Nervosität zu erreichen versucht wird. Bisher gab es nur Querschnittsuntersuchungen, die eine Einjahres-Einnahmehäufigkeit mittels anonymer Fragetechniken von bis zu $20 \%$ unter Studierenden zeigten [1]. Mit dem DAK-Report liegt erstmals eine Langzeitstudie vor, mit der auch die Frage beantwortet werden kann, ob Hirn-Doping in Deutschland zunimmt.

DAK-Gesundheitsreport 2015. Schwerpunktthema: Update Doping am Arbeitsplatz: pharmakologisches Neuroenhancement durch Erwerbstätige. S. $29-96$
Methodik: Das IGES-Institut befragte eine repräsentative Bevölkerungsstichprobe von 5.017 Erwerbstätigen (10.213 wurden angeschrieben, Rücklaufquote $49,1 \%$ ) zwischen 20 und 50 Jahren mittels anonymer Fragebögen. Um die Dunkelziffer zu ermitteln, wurden die Probanden auch mittels einer speziellen Fragetechnik, die Anonymität garantiert, befragt, der sogenannten Unmatched Count Technique (UCT).

Ergebnisse: 6,7\% der Berufstätigen hatten Hirn-Doping schon mindestens einmal praktiziert, während das 2008 noch $4,7 \%$ gewesen waren. Dabei wurde häufiger Enhancement zur Steigerung des Wohlbefindens (4,7\%) als zur Leistungssteigerung (3,3\%) betrieben. Die Dunkelziffer für die Einnahme lag bei bis zu $12 \%$, was hochgerechnet fünf Millionen dopende Beschäftigte in Deutschland bedeutet. Von den Konsumenten nehmen zirka $45 \%$ die Substanzen täglich ein, $63 \%$ nutzen sie alle zwei Wochen oder häufiger. Am häufigsten werden Substanzen gegen Angst, Nervosität und Unruhe (61 \%) sowie Medikamente gegen Depressionen (34\%) eingenommen. 12\% nehmen Substanzen gegen erhöhte Tagesmüdigkeit, $11 \%$ Betablocker, 9\% Substanzen gegen ADHS und $7 \%$ gegen Gedächtniseinbußen. Während Männer Substanzen eher zur Leistungssteigerung einsetzen, nutzen Frauen HirnDoping eher zur Stimmungsaufhellung und zum Abbau von Ängsten und Nervosität. Bezüglich demografischer Variablen wie Schulabschluss, Berufsstand (Arbeiter, Angestellte, Beamte etc.) und Akademiker versus Nichtakademiker gab es keine Unterschiede. Jedoch zeigten eher Beschäftigte mit einfacher Arbeit und unsicherem Arbeitsplatz ein erhöhtes Konsumrisiko.

Schlussfolgerungen: Pharmakologisches Neuroenhancement mit verschreibungspflichtigen Medikamenten hat seit $2008 \mathrm{zu}-$ genommen und stellt ein relevantes gesellschaftliches Phänomen dar.

\section{Pharmakologisches Neuroenhancement auf dem Vormarsch}

Der DAK-Report 2015 ist methodisch sehr gut gemacht und bringt einige überraschende Ergebnisse. Erstmals wurde von einer Arbeitsgruppe unter Verwendung gleicher oder sehr ähnlicher Fragen gezeigt, dass Hirn-Doping in Deutschland zunimmt. Überraschend ist, dass es offenbar nicht die Personen auf der Führungsebene sind, die Hirn-Doping betreiben, sondern eher Erwerbstätige mit einfacher Arbeit. Dennoch zeigt der Report, dass Motive, die man auch in der mittleren bis hohen Führungsebene häufig finden dürfte, häufige Doping-Motive sind, nämlich „bis an die Grenzen der Leistungsfähigkeit arbeiten“, „Prüfungen, Präsentationen und schwierige Gespräche besser meistern" und "Ziele besser erreichen“. Unsere Daten zur Dunkelziffer von 20\% für die Einnahme von Enhancern im letzten Jahr und die hohe grundsätzliche Bereitschaft von Studierenden Hirn-Doping zu betreiben, lassen vermuten, dass das Phänomen weiter an Bedeutung zunehmen wird. Verschreibungspflichtige Medikamente sind nicht umsonst verschreibungspflichtig, dazu kommen die hohen Placeboresponseraten etwa bei Antidepressiva oder auch Methylphenidat. Aufgrund dieser ungünstigen Nutzen-Schadens-Relation sollte von HirnDoping bei Gesunden abgeraten werden. Mehrere Studien zeigen, dass man mit Koffein vergleichbare Effekte erzielen kann [2]. Bleiben wir also besser beim Kaffee.
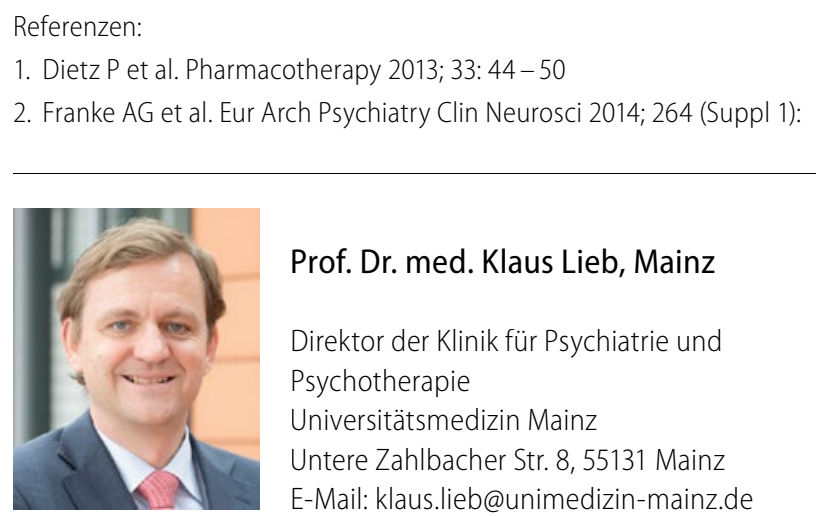

Prof. Dr. med. Klaus Lieb, Mainz

Direktor der Klinik für Psychiatrie und

Psychotherapie

Universitätsmedizin Mainz

Untere Zahlbacher Str. 8, 55131 Mainz

E-Mail: klaus.lieb@unimedizin-mainz.de 